Full Length Article

\title{
Recyclable magnetic graphene oxide for rapid and efficient demulsification of crude oil-in-water emulsion
}

\author{
Juan Liu ${ }^{\mathrm{a}, \mathrm{b}}$, Huanjiang Wang ${ }^{\mathrm{a}, \mathrm{b}}$, Xiaocheng Li $^{\mathrm{c}}$, Weihong Jia ${ }^{\mathrm{a}}$, Yapu Zhao ${ }^{\mathrm{d}}$, Sili Ren ${ }^{\mathrm{a}, *}$ \\ a State Key Laboratory of Solid Lubrication, Chinese Academy of Sciences, Lanzhou 730000, People's Republic of China \\ ${ }^{\mathrm{b}}$ University of Chinese Academy of Sciences, Beijing 100049, People's Republic of China \\ 'Laboratory of Clean Energy Chemistry and Materials, Chinese Academy of Sciences, Lanzhou 730000, People's Republic of China \\ d State Key Laboratory of Nonlinear Mechanics, Institute of Mechanics, Chinese Academy of Sciences, Beijing 100190, People's Republic of China
}

\section{H I G H L I G H T S}

- The M-GO was a high efficiency demulsifier which could be reused for 6-7 times.

- The non-covalent interaction analysis proved that $\pi-\pi$ and $\sigma-\pi$ interactions between GO materials and asphaltene molecules are the major driven forces for demulsification.

\section{A R T I C L E I N F O}

\section{Article history:}

Received 16 July 2016

Received in revised form 6 October 2016

Accepted 12 October 2016

Available online 25 October 2016

\section{Keywords:}

Magnetic graphene oxide

Demulsification

Crude oil-in-water emulsion

Recyclability

Non-covalent interaction analysis

\section{G R A P H I C A L A B S T R A C T}

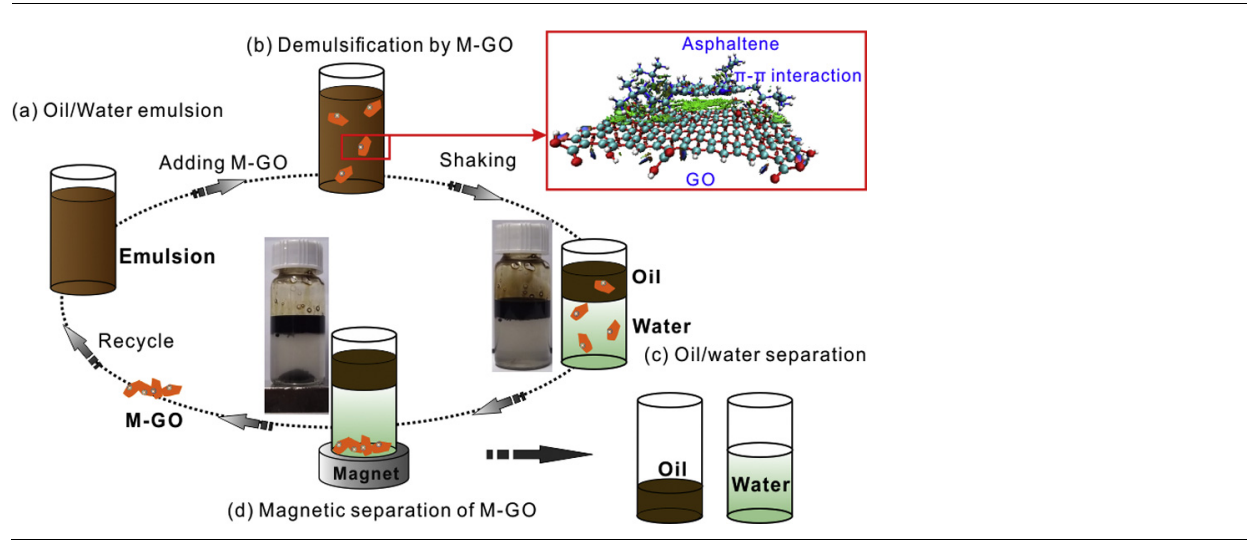

\section{A B S T R A C T}

Graphene oxide (GO) nanosheets have been experimentally proved to be a highly efficiency, rapid and universal demulsifier to break up the crude oil-in-water emulsion and/or the emulsified oily waste water in our previous study. To recycle the GO nanosheets and avoid the possible contamination of GO for crude oil, in this work, the magnetic graphene oxide (M-GO) was successfully synthesized and used for separating oil/water emulsions. Demulsification tests indicated that M-GO could separate the oil/water emulsions within a few minutes and recycle 6-7 times without losing its demulsification capability. The residual oil content in the separated water was as low as $\sim 10 \mathrm{mg} / \mathrm{L}$, corresponding to a demulsification efficiency of $99.98 \%$ at an optimal dosage. Quantum chemical calculation results indicated that the $\pi-\pi / \sigma-\pi$ interactions between GO materials and asphaltene molecules are the major driven forces for the high demulsification performance of M-GO nanosheets. This work not only provides a promising demulsifies to demulsify the crude oil-in-water emulsion or the oily wastewater but also give a deep understanding on the intrinsic interaction between demulsifiers and asphaltenes.

(c) 2016 Elsevier Ltd. All rights reserved.

\footnotetext{
* Corresponding author.

E-mail address: slren@licp.cas.cn (S. Ren).
}

\section{Introduction}

In order to meet the increasing energy demand, a host of policies has been made to encourage exploiting the unconventional oil resources including the heavy oil, oil sands and oil shale [1]. 
Water blending has become one of important strategies in many mining processes [2], such as the tertiary oil recovery, waterbased bitumen extraction processes [3] and heavy oil viscosity reduction technology [4]. These processes generally involved the exploiting and subsequent refinery operations, and would produce large amount of oil-in-water $(\mathrm{O} / \mathrm{W})$, water-in-oil (W/O) emulsions and/or emulsified oily wastewater. The produced oil-water emulsions are highly undesirable in petroleum industry because of their high corrosion to the pipe surface and distillation equipments, high toxicity to the catalysts in refining processes, and serious pollution problems to environment. High efficiency demulsification of oil-water emulsions through various strategies is therefore of significant importance for petroleum industry. Till now, the demulsification of $\mathrm{W} / \mathrm{O}$ emulsions has been considerably investigated and can be successfully realized by adding several types of chemical demulsifiers such as PAMAM-polyether [5], ethylene oxide/propylene oxide block copolymer [6], silicone surfactant [7], ethylcellulose polymer [8], alkoxylated alkyl phenol formaldehyde resin [9], and ionic liquid [10]. In contrast, the O/W emulsion has been one of worldwide challenges because of its increasing production from the oilfields and the serious contaminations resulted from oily wastewater [11]. Considerable efforts were thereby devoted to demulsify the $\mathrm{O} / \mathrm{W}$ emulsions. The oil-water mixture can be classified into two types, namely, the oil-water suspension with free or dispersed oil droplet $(\mathrm{d}>20 \mu \mathrm{m})$ suspended in water and $\mathrm{O} / \mathrm{W}$ emulsion with oil droplet size of $\mathrm{d}<20 \mu \mathrm{m}$. The former oil-water suspension, can be easily separated through the gravity separation, centrifugation, ultrasonic strategies and/or employment of superhydrophobic and superoleophilic adsorbent materials. While for the latter $\mathrm{O} / \mathrm{W}$ emulsion, due to the small size of oil droplet and highly stable oil-water interface protective film, it is more difficult to separate the oil droplet from O/W emulsion. Attempts have been carried out to separate the oily wastewater by using membrane or filtration technology [12-16]. However, these techniques still have disadvantages of low separation efficiency, long separation time, and usage of special equipments with high cost and extremely large size. Moreover, these techniques would generate secondary pollutants, fouling problems, and poor regeneration/recyclability, limiting their practical application in oil/water separation [17]. Therefore, it is necessary to develop a simple but effective method for separating O/W emulsions with high separation efficiency, short separation time.

In recent study, we develop a simple but effective demulsification method for fast separating the $\mathrm{O} / \mathrm{W}$ emulsions by using grapheme oxide (GO) nanosheets as demulsifier [18]. The developed GO demulsifier demonstrates a demulsification efficiency of 99.9\% and extremely low residual oil content $(30 \mathrm{mg} / \mathrm{L})$ in separated water at a lower dosage of $20-100 \mathrm{mg} / \mathrm{L}$. Moreover, the whole demulsification process can be completed within several minutes. Despite the fast and high separation efficiency, after separation, the GO demulsifier stably exist in aqueous or oil phase in the form of nanosheets, which either would bring the contamination to water or oil in the subsequent refinery operation. Therefore, some measurements must be taken to recycle the GO nanosheets to minimize the contamination of GO and simultaneously reduce the demulsification cost.

Magnetic nanoparticles (MNPs) are of great interest for researchers due to their applications in most fields, such as biotechnology/biomedicine, catalysis, contrast enhancement of magnetic resonance imaging, environmental remediation and magnetic separation [19]. For example, anchoring the MNPs $\left(\gamma-\mathrm{Fe}_{3} \mathrm{O}_{4}\right.$ and $\left.\gamma-\mathrm{Fe}_{2} \mathrm{O}_{3}\right)$ on host materials would endow them with excellent magnetism for magnetic separation. This strategy has been successfully used to separate host materials from the complex mixtures, such as the biomaterials [20] including cell, bacteria, protein, DNA, wastewater [21] and emulsions [19,22].
Inspired by the successful applications of MNPs in magnetic separation, in present study, we attempt to decorate the GO nanosheets with MNPs to recycle the GO nanosheet after the demulsification tests with the aid of external magnetic field. To prevent the selfaggregation of MNPs and increase their anti-corrosion performance in different chemical, before grafting to GO nanosheets, a thin layer of silica film was deposited on MNPs surface to stabilize them and provide a desirable surface for further aminofunctionalization. Through the chemical reaction between the functional groups on GO nanosheets and amino-group on silicastabilized MNPs, we successfully synthesize the silica-stabilized magnetic $\gamma-\mathrm{Fe}_{2} \mathrm{O}_{3}-\mathrm{GO}$ composite (M-GO) for demulsificating of $\mathrm{O} /$ $\mathrm{W}$ emulsions. The developed M-GO could separate the oil/water emulsions within a few minutes and demonstrate a high demulsification efficiency of $99.98 \%$ with an extremely low residual oil content of $\sim 10 \mathrm{mg} / \mathrm{L}$ in separated water. More importantly, the M-GO can be recycled within few seconds under an external magnetic field and repeated usage for 6-7 times without losing its demulsification capability, suggesting a fast a low-cost demulsification method.

\section{Materials and methods}

\subsection{Materials}

Graphite (synthetic powder, $<20 \mu \mathrm{m}$, Sigma-Aldrich), (3-Aminopropyl) triethoxysilane (3-APTES, Aladdin Reagent, Shanghai, China), 1-(3-dimethylaminopropyl)-3-ethylcarbodii mide hydrochloride (EDC.HCl, GL Biochem, Shanghai, China), and N-Hydroxy succinimide (NHS, Kefeng Chemical Reagent, Shanghai, China) were directly used without further treatment. The crude oil sample was provided by Tahe oilfield (Xingjiang Province, China). The physiochemical properties of the oil sample were reported in our previous work [18]. The iron carbonyl $\left(\mathrm{Fe}(\mathrm{CO})_{5}\right)$ (Zhongke Haorong New Material Research Co., Ltd., Changchun, China) were used to prepare magnetic ferrite. The Kerosene was obtained from local gas station of Sinopec (Lanzhou, China). Ultrapure water $(18.25 \mathrm{M} \Omega \mathrm{cm})$ was used throughout the experiments.

\subsection{Preparation of $M-G O$}

The synthesis processes of M-GO by a covalent strategy were illustrated in Fig. 1(a). In the process, the iron carbonyl was used to prepare magnetic ferrite by using the oxidization/decomposition method. The magnetic ferrite was then coated with a layer of silica to improve the chemical stability and to make the surface amenable for further functionalization. The silica shell was subsequently modified with organic silane coupling agent (3-APTES) to render a surface of amine groups. Finally, the GO nanosheets (prepared by a modified Hummers method [23]) reacted with the amine groups on the amino-functionalized magnetic particles to form the magnetic GO nanosheets (the details of preparation and characterization of various magnetic nanoparticles are given in the Supplementary Material).

\subsection{Preparation of the crude oil-in-water emulsion}

Tahe crude oil sample was directly used without further treatment. The crude oil-in-water emulsion was prepared by mixing crude oil with brine water $(50 \mathrm{mmol} / \mathrm{L} \mathrm{NaCl})$. Briefly, $20 \mathrm{~g}$ of oil was added to $380 \mathrm{~g}$ brine water with $\mathrm{pH}$ of $5.7,4$, or 2 in a plastic bottle. The mixture was emulsified by using an ultrahigh speed homogenizer (Fluko, FA25) operated at 28,000 rpm for $5 \mathrm{~min}$ to form a crude oil-in-water emulsion oil content of $5.0 \mathrm{wt} \%$. 

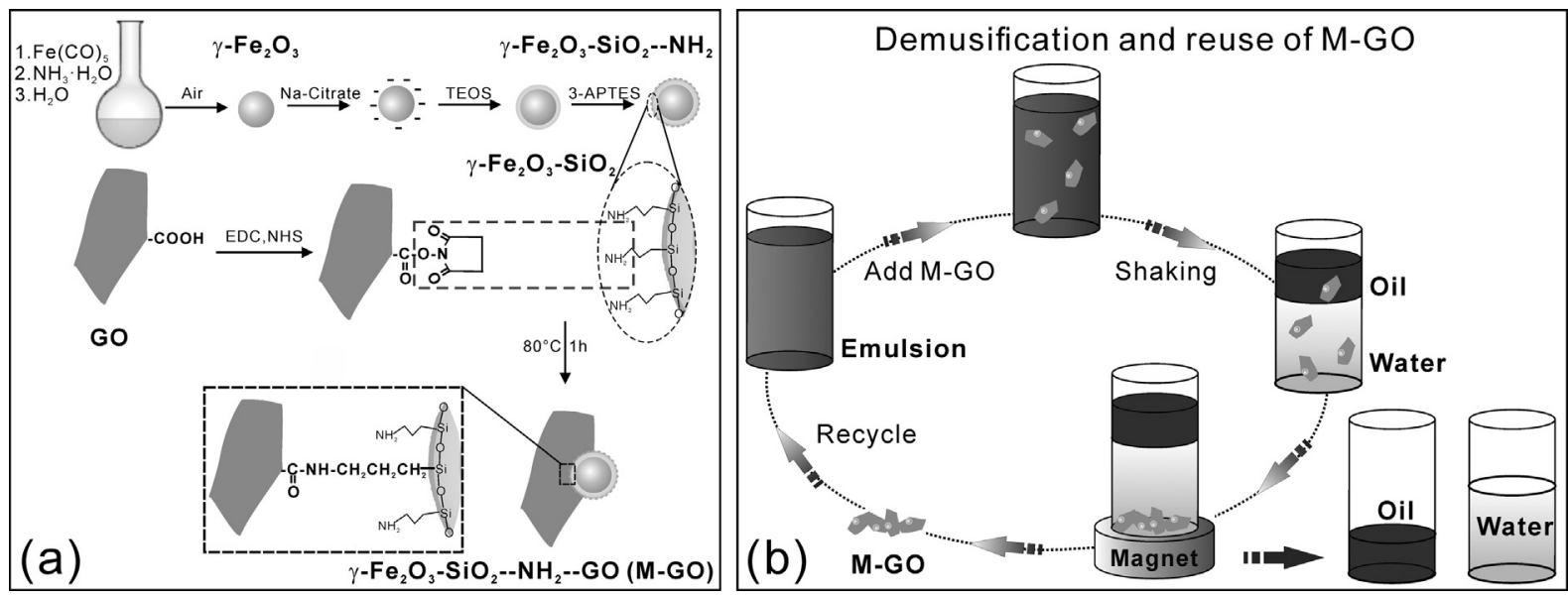

Fig. 1. (a) Preparation of the magnetic graphene oxide and (b) schematic illustration of demulsification and recycling tests using M-GO as a demulsifier.

\subsection{Demulsification test}

The procedure of demulsification test was shown in Fig. 1(b). In each test, proper amount of M-GO aqueous suspension (1.0 wt\%) after shaking was added to the as-prepared emulsion. The dosage of M-GO can be calculated based on the mass concentration. After thoroughly shaking, the mixture was then allowed to settle under ambient condition to observe the oil/water separation. The emulsion without any addition was employed as the reference (blank). When the oil phase separated from the water, a magnet was used to accelerate the separation of residual oil floccules in water. Then, the separated water samples were subsequently separated from the newly formed oil phase scrupulously by using a separatory funnel.

The demulsification efficiency of M-GO was determined by measuring the residual oil content in the separated water sample. The residual oil was extracted by kerosene three times and the oil content which has a linear relation with the absorbance of kerosene-diluted-oil was analyzed by UV-visible Spectrophotometer (Unico, UV-2000) at the wavelength of $350 \mathrm{~nm}(1 \mathrm{~cm}$ path length). Each test was repeated three times and the average values were reported. The oil concentration in the separated water samples and the demulsification efficiency were calculated by the equations:

$c=\frac{m_{0}}{V_{w}} \times 10^{3}$

$E=\frac{c_{0}-c_{i}}{c_{0}} \times 100 \%$

where $m_{0}$ is the mass of oil, $V_{w}(\mathrm{~mL})$ is the volume of the water samples; $c(\mathrm{mg} / \mathrm{L})$ is the oil concentration, $c_{0}$ or $c_{i}$ is the oil concentration of emulsion or separated water. The demulsification efficiency $(E)$ of $\mathrm{M}-\mathrm{GO}$ is the percentage of oil removed from the emulsion. Obviously, the higher the $E$ is, the better the demulsification performance will be.

The micro morphology of the emulsion, the separated water and oil phases was observed by using a polarizing microscope (Leica, DM2500P) equipped with a digital camera. Various specimens for the observation were prepared by dripping them onto a clean glass slide $(25 \times 75 \times 0.2 \mathrm{~mm})$ and then covered with another one.

\subsection{Recycling test}

To evaluate the recyclability of the magnetic demulsifier, the MGO was collected and separated by using a magnet after demulsi- fication test. The recycled M-GO was then repeatedly washed by toluene for several times to wash off the adsorbed asphaltene molecules on the surface of M-GO. As the supernatant become colorless, the M-GO was collected and dried under vacuum at $60^{\circ} \mathrm{C}$, and then used for the following demulsification test, which was considered as one time recycle. In this work, the recycles test was performed eleven times (the test was finished when the oil concentration in the separated water was $>500 \mathrm{mg} / \mathrm{L}$ ).

\subsection{Quantum chemical calculations}

The non-covalent interactions between $\mathrm{GO}\left(\mathrm{C}_{47} \mathrm{H}_{38} \mathrm{O}_{22}\right)$ and asphaltene molecules (modified Yen model [24]) were performed with the ORCA-3.0.3 program package using the dispersion corrected BLYP-D3 functional together with the double- $\zeta$ Def2-SVP basis set. The geometries of GO, asphaltenes and GO-asphaltene interaction pair were optimized in vacuum.

The non-covalent interaction analysis has been carried out with the Multiwfn program utilizing either its default parameters or a uniform spatial grid with a step of 0.1 a.u., respectively. The noncovalent interaction regions are then visualized using Visual Molecular Dynamics software version 1.9.1.

\section{Results and discussion}

\subsection{Characterization of various nanoparticles}

Fig. 2(a) shows the typical atomic force microscopy (AFM) image of exfoliated GO nanosheets. It can be observed that the GO with size of $100-1500 \mathrm{~nm}$ and thickness of $\sim 1.2 \mathrm{~nm}$ (Fig. 2 (b)) are uniformly distributed on the silicon surface. The asprepared GO consisting of one or two layers of the nanosheets were obtained in this work.

In this study, the MNPs were prepared as the magnetic core. The $\mathrm{X}$-ray diffraction (XRD) measurements were employed to investigate the phase and structure of the as-prepared MNPs. As shown in Fig. 2(c), several well-defined diffraction peaks were clearly observed at $2 \theta$ value of $30.24^{\circ}, 35.64^{\circ}, 43.21^{\circ}, 53.41^{\circ}, 57.26^{\circ}$, and $62.85^{\circ}$, which can be indexed as the (220), (311), (400), (422), (511) and (440) plane reflections of $\gamma-\mathrm{Fe}_{2} \mathrm{O}_{3}$ (JCPDS card 391346) [25]. The fitted lattice constant of the unit cell dimension was $8.351 \AA$, which is good agreement with cubic $\gamma-\mathrm{Fe}_{2} \mathrm{O}_{3}$ [26]. The chemical composition of the magnetic particles was further confirmed by X-ray photoelectron spectrum (XPS) analysis of the Fe2p orbital (Fig. 2(d)). In particular, the $\mathrm{Fe} 2 \mathrm{p}_{3 / 2}$ binding energy peak appears at $710.7 \mathrm{eV}$, which is close to the characteristic peak of the electronic structure of $\mathrm{Fe}^{3+}$ in $\gamma-\mathrm{Fe}_{2} \mathrm{O}_{3}$ [27]. Based on the 

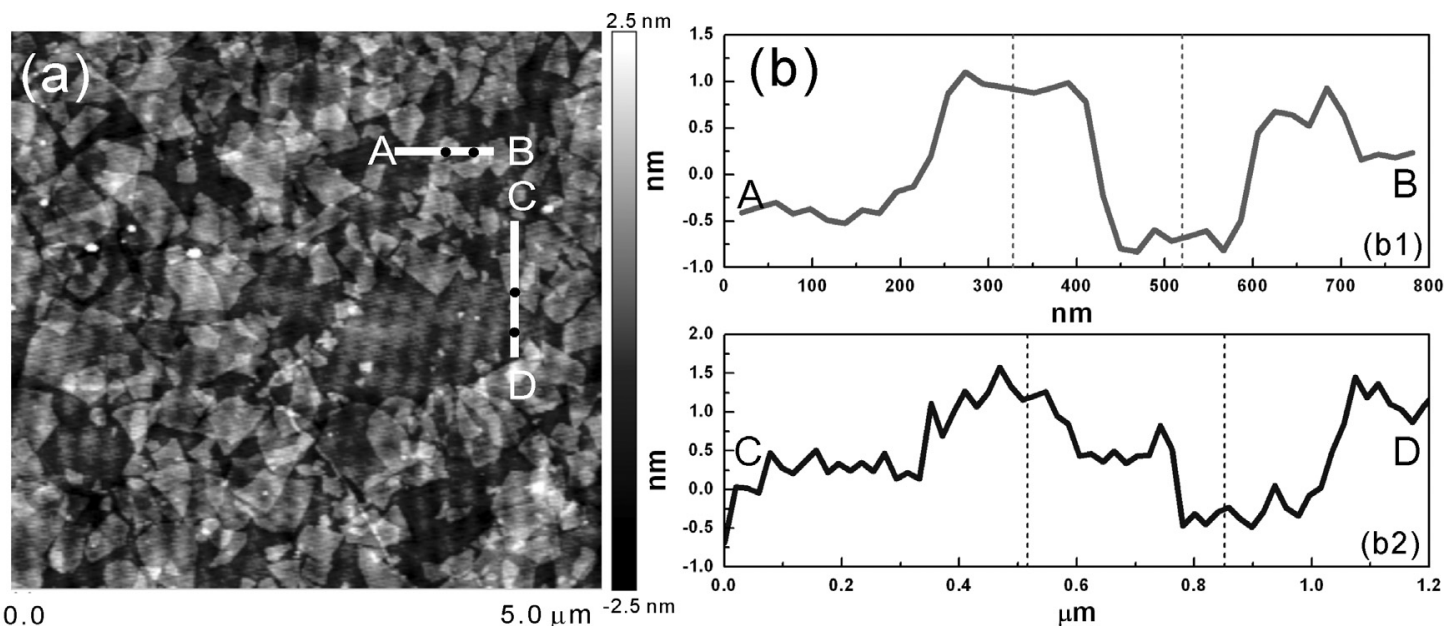

0.0

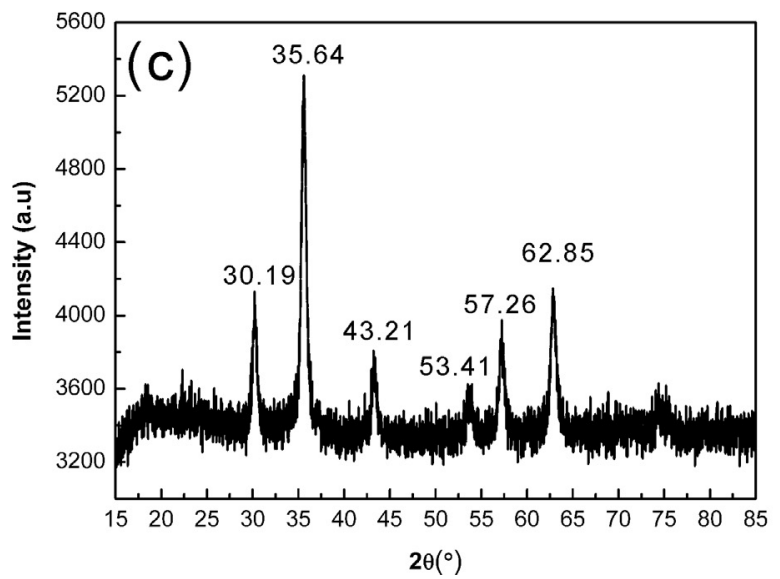

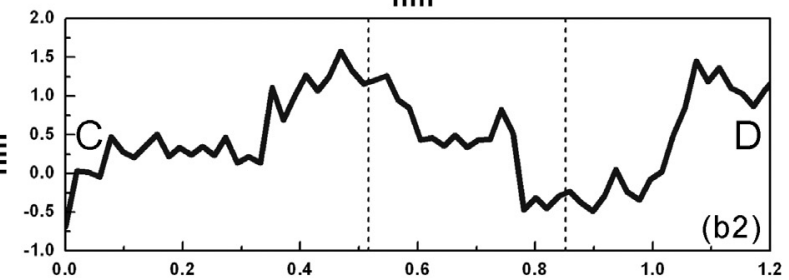

$\mu \mathrm{m}$

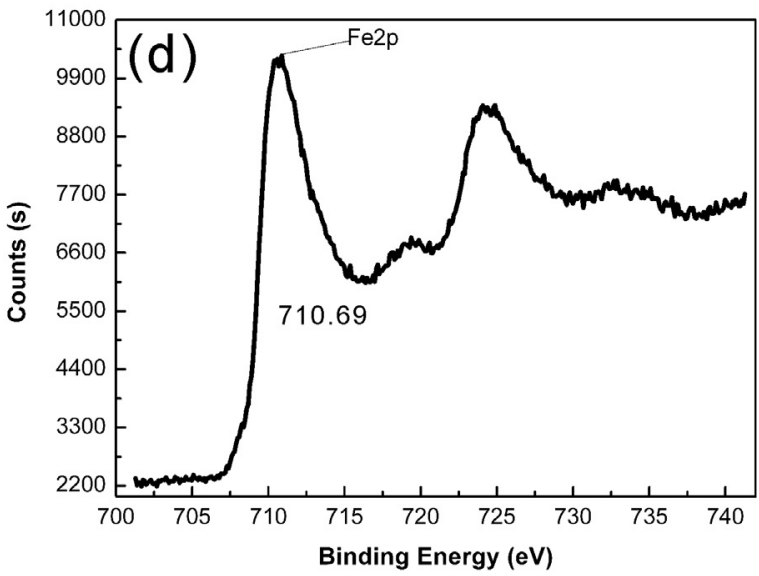

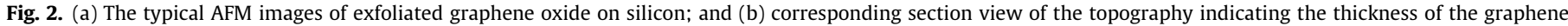
oxide nanosheets. (c) The XRD pattern and XPS spectrum (d) of the as-prepared $\gamma-\mathrm{Fe}_{2} \mathrm{O}_{3}$ nanoparticles.

results of XRD and XPS, it can be concluded that the prepared magnetic ferrite was $\gamma-\mathrm{Fe}_{2} \mathrm{O}_{3}$.

The typical high resolution transmission electron microscopy (HRTEM) images of bare $\gamma-\mathrm{Fe}_{2} \mathrm{O}_{3}, \quad \gamma-\mathrm{Fe}_{2} \mathrm{O}_{3} @ \mathrm{SiO}_{2}$ (core-shell nanoparticle) and M-GO were shown in Fig. 3(a). It was observed that the $\gamma-\mathrm{Fe}_{2} \mathrm{O}_{3}$ nanoparticles aggregated because of their magnetism (Fig. 3(a1)). The average diameter of $\gamma-\mathrm{Fe}_{2} \mathrm{O}_{3}$ nanoparticles is $\sim 10 \mathrm{~nm}$. After being coated with nonporous silica layer, nearspherical core-shell nanoparticles of $\gamma-\mathrm{Fe}_{2} \mathrm{O}_{3} @ \mathrm{SiO}_{2}$ were obtained (Fig. 3(a2)). Obviously, the black $\gamma-\mathrm{Fe}_{2} \mathrm{O}_{3}$ particles were wrapped by a silica layer with thickness of $\sim 50 \mathrm{~nm}$ (light gray). As shown in Fig. 3(a3), the amino-functional MNPs with the average diameter of $\sim 100 \mathrm{~nm}$ were successfully anchored on the surface of GO, in which the GO was thin and transparent and looked like the silk veil waves.

Zeta potential of various nanopaticles collected from each stage of preparation were determined in the $1 \mathrm{mM} \mathrm{KCl}$ aqueous solutions with pH of 5.3 [28]. As shown in Fig. 3(b1), the $\gamma-\mathrm{Fe}_{2} \mathrm{O}_{3}$ nanoparticles were positive charged with the average zeta potential value of $11.48 \mathrm{mV}$. While the zeta potential of $\gamma-\mathrm{Fe}_{2} \mathrm{O}_{3} @ \mathrm{SiO}_{2}$ became negative of $-44.87 \mathrm{mV}$, which is good agreement with that of silica at the same condition [28,29]. The result confirmed that the $\gamma$ $\mathrm{Fe}_{2} \mathrm{O}_{3}$ nanoparticles were fully coated by silica. The zeta potential of silanized $\gamma-\mathrm{Fe}_{2} \mathrm{O}_{3} @ \mathrm{SiO}_{2}$ particles by 3-APTES became positive again $(\sim 7.14 \mathrm{mV})$ due to the protonation of amino groups on the silica surface. Such reverse confirmed that the 3-APTES molecules have been successfully grafted to the silica surface. The exposed amino groups on the functionalized nanoparticles $\left(\gamma-\mathrm{Fe}_{2} \mathrm{O}_{3} @ \mathrm{SiO}_{2}-\right.$
$\mathrm{NH}_{2}$ ) surface are the active sites to react with GO. After coupling the amino-functionalized nanoparticles on the GO surface, the zeta potential of M-GO were measured to be $-35.61 \mathrm{mV}$, which was slightly smaller than that of GO (range from -42 to $50 \mathrm{mV}$ [30]) due to the influence of acylamino groups and the unreached amino groups on the silica surface. These results from the zeta potential measurements provide convincing evidence that the anticipated nanoparticles were successfully obtained in each step during the preparation of M-GO.

To confirm the chemical structure, various nanopaticles were characterized by a Fourier transform infrared spectrometer (FTIR). As depicted in Fig. 3(b2), the bare $\gamma-\mathrm{Fe}_{2} \mathrm{O}_{3}$ has predominant peaks at $\sim 3400 \mathrm{~cm}^{-1}$ and $\sim 576 \mathrm{~cm}^{-1}$, which were attributed to the vibration of $\mathrm{Fe}-\mathrm{OH}$ and $\mathrm{Fe}-\mathrm{O}$, respectively. The peak at $2962 \mathrm{~cm}^{-1}$ and $2930 \mathrm{~cm}^{-1}$ was the characteristic of $\mathrm{C}-\mathrm{H}$ stretching mode due to the existence of residual ethylenediamine in preparation of the $\gamma-\mathrm{Fe}_{2} \mathrm{O}_{3}$ nanoparticles. When the $\gamma-\mathrm{Fe}_{2} \mathrm{O}_{3}$ nanoparticles were treated by TEOS, the peak at $\sim 3734 \mathrm{~cm}^{-1}$ was observed, which was assigned to the vibration of $\mathrm{Si}-\mathrm{OH}$ of the coated silica. After silane treatment of the $\gamma-\mathrm{Fe}_{2} \mathrm{O}_{3} @ \mathrm{SiO}_{2}$ by 3-APTES, the peaks centered at $2963 \mathrm{~cm}^{-1}$ and $2925 \mathrm{~cm}^{-1}$ became stronger than that of $\gamma-\mathrm{Fe}_{2} \mathrm{O}_{3}$ and $\gamma-\mathrm{Fe}_{2} \mathrm{O}_{3} @ \mathrm{SiO}_{2}$, which were corresponding to the stretching vibration of $-\mathrm{CH}_{2}$ groups of aminopropyl from 3APTES, indicating that the 3-APTES successfully grafted to the surface of $\gamma-\mathrm{Fe}_{2} \mathrm{O}_{3}-\mathrm{SiO}_{2}$. However, the stretching vibration of amino groups was not observed, which might hide behind the broad bands of hydroxyl group. For the spectrum of M-GO, the strong peaks at $3422 \mathrm{~cm}^{-1}, 1720 \mathrm{~cm}^{-1}, 1618 \mathrm{~cm}^{-1}$ and $1050 \mathrm{~cm}^{-1}$ were 

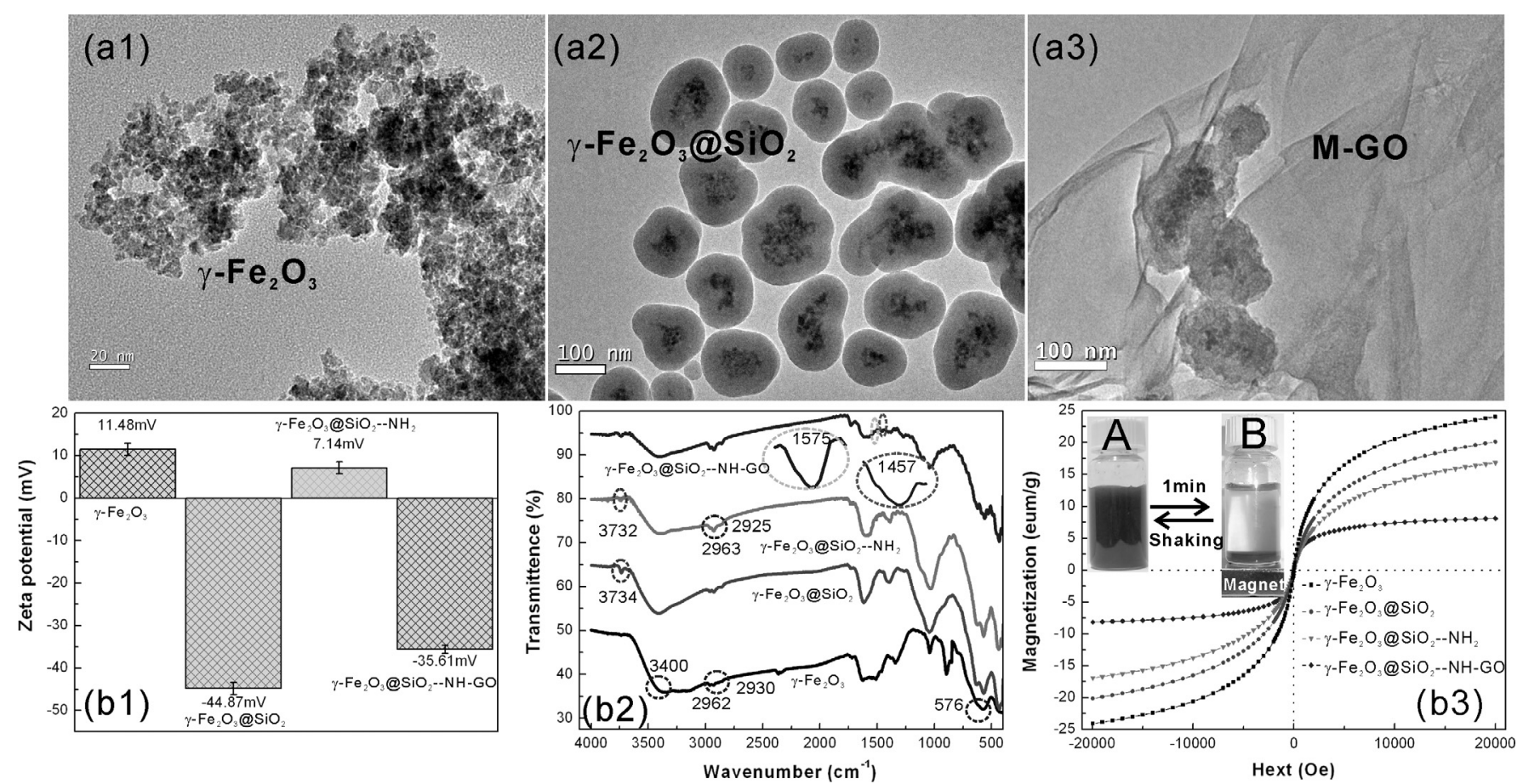

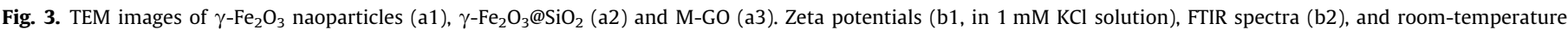

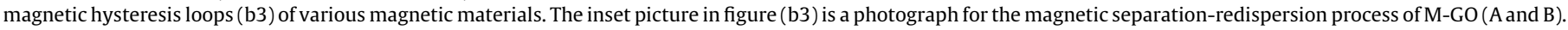

the characteristic vibration of hydroxyl groups $(-\mathrm{OH})$, carbonyl groups $(-\mathrm{C}=\mathrm{O})$, and alkoxy groups $(\mathrm{C}-\mathrm{O}-\mathrm{C})$ of $\mathrm{GO}$ [31]. The small peak at $\sim 1575 \mathrm{~cm}^{-1}$ or $\sim 1457 \mathrm{~cm}^{-1}$ was attributed to the deformation vibration of $\mathrm{N}-\mathrm{H}$ or the stretching vibration of $\mathrm{C}-\mathrm{N}$ in the amide group ( $\mathrm{CO}-\mathrm{NH}-$ ), signifying that the amino-functional magnetic particles chemically anchored on the surface of GO.

For application of the magnetic demulsifiers to break up the crude oil-in-water emulsion, it is important that the MNPs possess the magnetic properties so that they can be effectively recovered from the complex multi-phase system after demulsification. Therefore, the magnetic property of various MNPs was characterized. As shown in Fig. 3(b3), the hysteresis loops of various samples confirmed that the prepared MNPs possessed good superparamagnetism. The saturation magnetization of the $\gamma-\mathrm{Fe}_{2} \mathrm{O}_{3}, \gamma$ $\mathrm{Fe}_{2} \mathrm{O}_{3} @ \mathrm{SiO}_{2}, \gamma-\mathrm{Fe}_{2} \mathrm{O}_{3} @ \mathrm{SiO}_{2}-\mathrm{NH}_{2}$ and $\mathrm{M}-\mathrm{GO}$ particles gradually decreased due to the coated silica and other functionalized groups. Although the saturation magnetization for M-GO was relative lower and about $8.7 \mathrm{emu} / \mathrm{g}$, it still had a good response to the external magnetic field. As shown in the inset picture of Fig. 3 (b3), the M-GO could be well attracted to the bottom from their homogeneous suspension by an external magnetic field, while redispersion occurred rapidly with a slight shaking once the magnetic field was removed. These results indicated that the M-GO has good magnetic responsivity and redispersibility, which are important for their practical application.

\subsection{Demulsification of the crude oil-in-water emulsion}

Bottle test method was employed to evaluate the demulsification performance of M-GO as a demulsifier to break up the stability of crude oil-in-water emulsion (Fig. 4(a)). To clearly display the demulsification efficacy, the fresh crude oil-in-water emulsion without addition of the demulsifier was used as a control (Fig. 4 (a1)), which was stable for at least $12 \mathrm{~h}$ without obvious phase separation. After introducing a small dosage of M-GO suspension, the color of emulsion was immediately changed, indicating the stability of the emulsion was destroyed. With the help of mechanical vibration, large oil droplets or floccules were quickly formed and floated on the top of the water to form the oil phase, realizing the separation of oil from the water (Fig. 4(a2)). The newly formed water phase exhibited light yellow color, which might attribute to the small oil floccules suspended in the water.

It is well known that the sedimentation of small oil floccules in the separated water by gravity is time consuming. To save time, the sedimentation of the can be enhanced by using a magnet. In this way, the water phase became colorless within $\sim 5 \mathrm{~min}$. In addition, due to the strong interaction between M-GO and oil, most of the magnetic demulsifier wrapped by oil was brought into the oil phase after demulsification. Encouragingly, as shown in Fig. 4(b), the spent M-GO could be easily recovered from the newly formed oil phase by using a magnet. The isolated M-GO could be reactivated by washing with toluene and reused for the demulsification.

To have a better understanding of the demulsification process, the micro-morphologies of the oil/water mixture before and after demulsification were observed by using a polarizing microscope. For the crude oil-in-water emulsion (5.0 wt\%), it was observed that the oil droplets with size of 3-15 $\mu \mathrm{m}$ homogeneously distributed in the water phase (Fig. 5(a)). After demulsification, small amount of water droplets with size of $10-500 \mu \mathrm{m}$ stayed in the newly formed oil phase (Fig. 5(b)). Moreover, aggregates of the M-GO were also found at the oil/water interface. For the separated water phase, there were some floccules composed of small oil drops and M-GO aggregates suspending in the water (Fig. 5(c)), which can be easily removed with an external magnetic field (Fig. 5(d)). The elimination of fine oil floccules would lead to an evident decrease of the oil content in the separated water. These results demonstrated that the M-GO is an excellent demulsifier to break up the crude oil-in-water emulsion.

\subsection{Effect of the M-GO dosage on the demulsification efficiency}

The demulsification efficiency of the M-GO was determined by the residual oil in the separated water. As shown in Fig. 6(a), the residual oil concentration in the separated water reduced to 

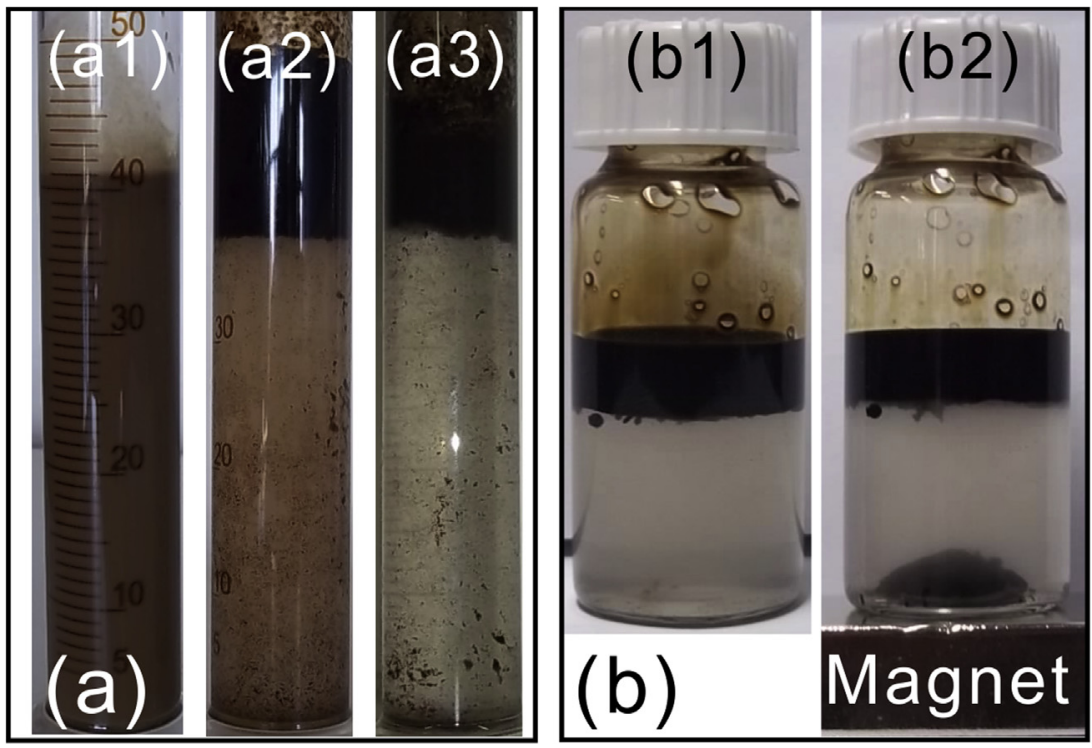

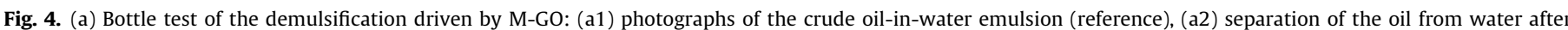

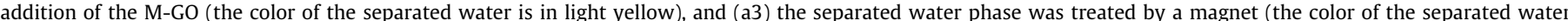

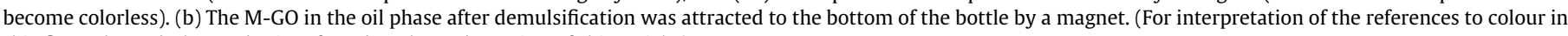
this figure legend, the reader is referred to the web version of this article.)
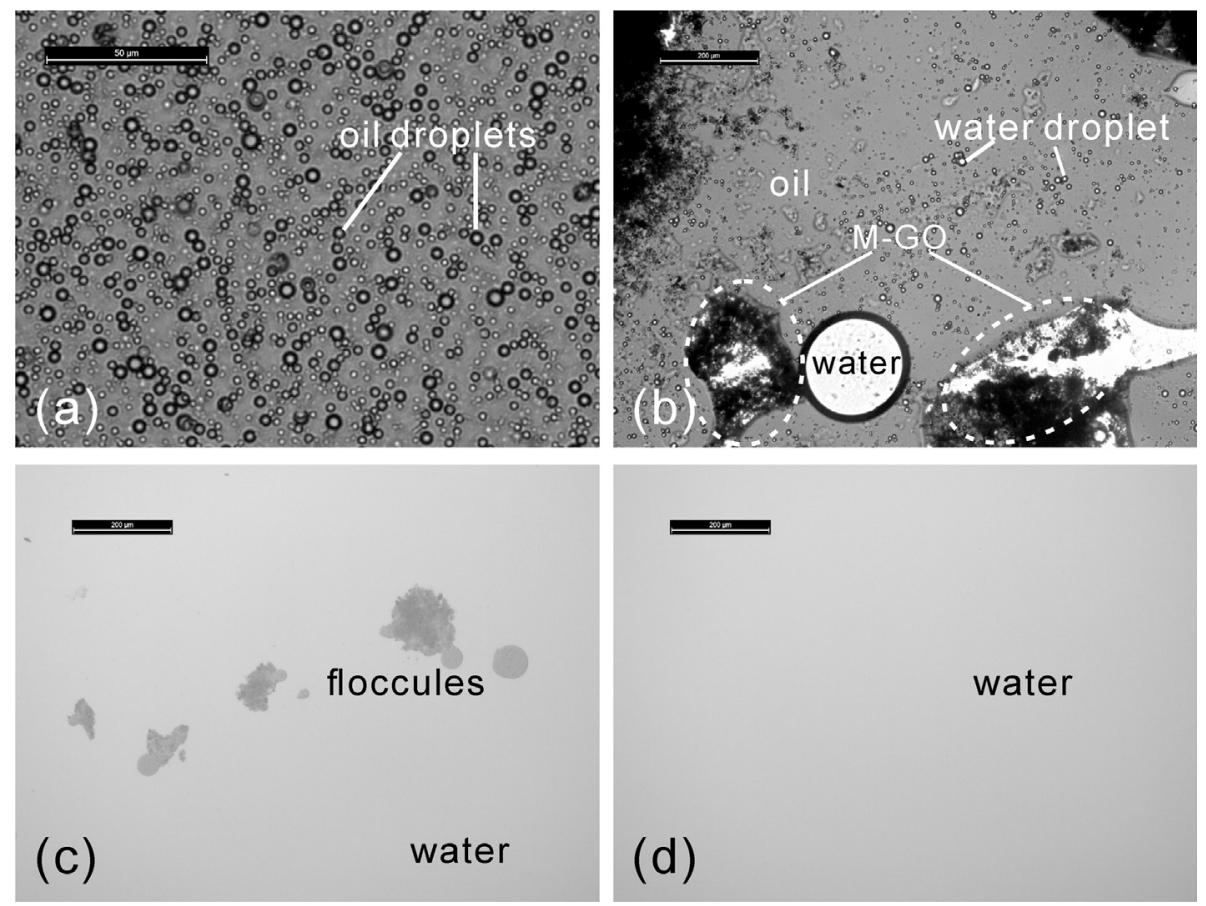

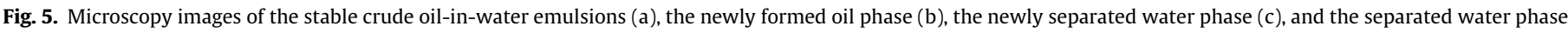
after treating with a magnet $(\mathrm{d})$.

$841.8 \pm 30 \mathrm{mg} / \mathrm{L}$ corresponding to a demulsification efficiency of about $98.3 \%$ with $0.04 \mathrm{wt} \% \mathrm{M}-\mathrm{GO}$ addition. It was greatly decreased with increasing the M-GO dosage. As the M-GO dosage increased to $0.25 \mathrm{wt} \%$, the residual oil concentration was reduced to $\sim 10 \mathrm{mg} / \mathrm{L}$ corresponding to a demulsification efficiency of as high as 99.98\%. Such an excellent demulsification performance of the M$\mathrm{GO}$ is much better than that of the GO reported in a previous study [18], which attribute to the fact that the very small oil floccules combined with M-GO suspending in the separated water after demulsification can be eliminated by external magnetic field.

\subsection{Effect of $\mathrm{pH}$ on the demulsification performance}

The hydrophilic edges and hydrophobic basal plane of the MGO enable it a good amphiphilicity. It is well known that the surfactivity of the ionic surfactant can be easily affected by the $\mathrm{pH}$ of the solution. Therefore, effect of the emulsion $\mathrm{pH}$ on the demulsification efficiency was investigated. It was found that the M-GO maintained a good demulsification performance in the acid condition. As shown in Fig. 6(b), the lowest oil concentration in the separated water was $<10 \mathrm{mg} / \mathrm{L}$ at $\mathrm{pH}$ of 2 or 4 . In addition, the 


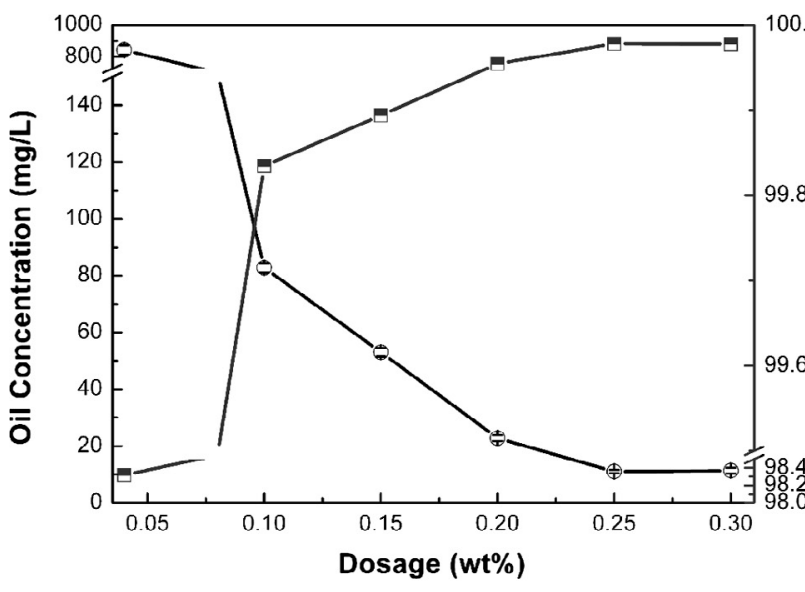

(a)
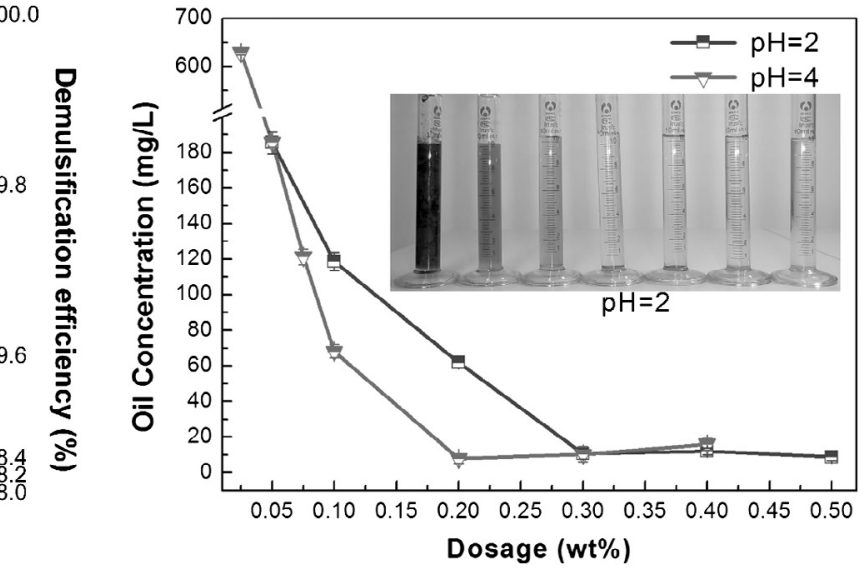

(b)

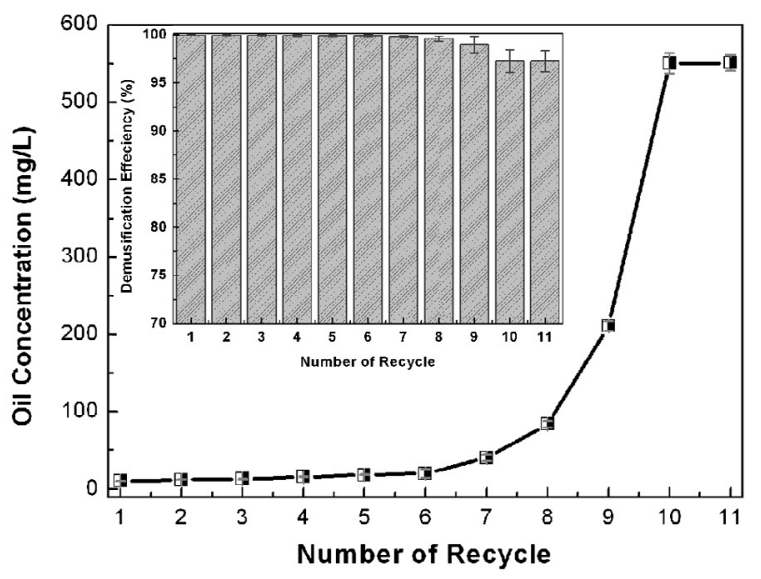

(c)

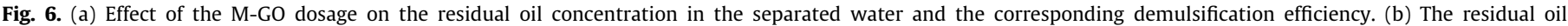

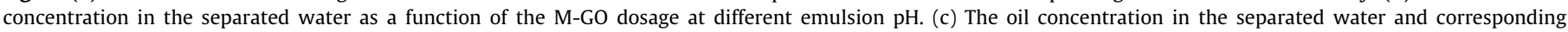
demulsification efficiency for the recycling demulsification tests.

separated water was almost colorless as the M-GO dosage was over $0.2-0.3 \mathrm{wt} \%$ (the inset picture in Fig. $6(\mathrm{~b})(\mathrm{pH}=2)$ ), indicating that there was very little of the oil floccules remaining in the separated water. Such a finding implied that the M-GO was rather entered into the oil phase after demulsification. This might attribute to the ionization of the functional groups of $-\mathrm{OH}$ and $-\mathrm{COOH}$ on the M-GO in the acid condition, which makes the M-GO less hydrophilic.

\subsection{Mechanism on the demulsification process driven by $M-G O$}

In our previous study, the mechanism on demulsification of the oil-in-water emulsion by GO was discussed [18]. However, how GO interacts with the asphlatenes and/or resins consisting of the cortical protective film at the oil/water interface is still not very clear. To identify the intrinsic interaction between $\mathrm{GO}$ and asphaltene molecules, non-covalent interaction $(\mathrm{NCI})$ analysis in real space was employed based on the electron density $(\rho)$ and its derivatives. For instance, the first derivative is $s(\rho)=\frac{1}{2\left(3 \pi^{2}\right)^{1 / 3}} \frac{|\nabla \rho|}{\rho^{4 / 3}}$, where $\nabla$ is gradient operator, and $|\nabla \rho|$ is the norm of electron density gradient $[32,33]$. Generally, the non-covalent interaction can be isolated as regions with low density and low reduced gradient. However, different types of interactions (i.e., hydrogen-bonding) may appear in the same region of density/reduced gradient space. To distin- guish these interactions, the second derivative of the density was considered. The sign of the second eigenvalue $\left(\lambda_{2}\right)$ of the electron-density Hessian matrix can be used to distinguish the bonded interactions $\left(\lambda_{2}<0\right)$ from non-bonded $\left(\lambda_{2}>0\right)$ interactions. Analysis of the sign of $\lambda_{2}$ would help to discern different types of non-covalent interactions, and the density itself provides information about their strength. Thus, the interaction types can be understood by the values of $\operatorname{sign}\left(\lambda_{2}\right) \rho$.

The gradient isosurfaces are colored according to the sponding values of $\operatorname{sign}\left(\lambda_{2}\right) \rho$, which is found to be a good indicator of the interaction strength. Generally, large and negative value of sign $\left(\lambda_{2}\right) \rho$ indicates an attractive interaction (such as dipole-dipole or hydrogen bonding), while a large and positive value implies that the interaction is nonbonding. Values near zero indicate a very weak interaction such as the van der Waals force. Fig. 7 displays the gradient isosurfaces for the interactions between GO and an asphaltene molecule. It is believed that the area located at the interlayer space between GO and asphaltene (the green color in Fig. 7) is nonbonding interaction corresponding to the $\pi-\pi$ interaction, while the overlapping regions between the aliphatic side chains of asphaltene and the hexagonal rings of GO is attributed to the $\sigma-\pi$ interaction. Due to the introduction of oxygenic groups (such as alkoxy groups, carbonyl and hydroxyl groups) on the hexagonal rings of $\mathrm{GO}$, repulsive interaction between the oxygenic groups and aromatic rings of asphaltene (the light red color 


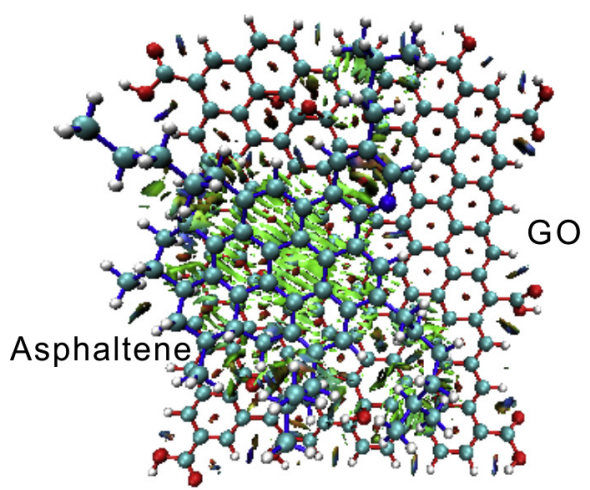

Top view (a)

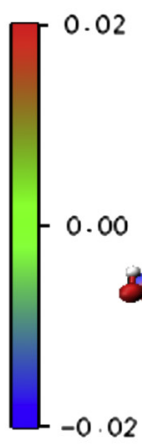

Side view

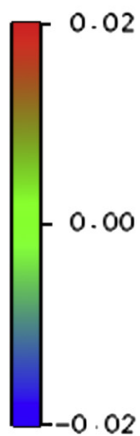

(b)

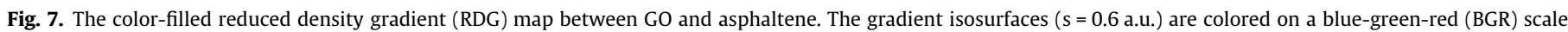

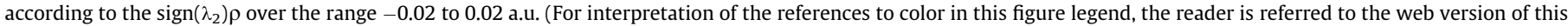
article.)

between GO and asphaltene interlayer space in Fig. 7(a)) was produced. As a result, the continuous $\pi-\pi$ interaction between GO and asphaltene was partially broken and the strength of $\pi-\pi$ interaction slightly decreased. Nevertheless, the $\pi-\pi$ and $\sigma-\pi$ interactions are still strong enough for the GO interacting with the asphlatenes.

As an amphiphile, the M-GO could well disperse in water and oil phase. Once it reaches at the oil/water interface to interact with the molecules of asphaltenes and/or resins, the protective film stabilizing the emulsion would be destroyed. As a result, small oil droplets would be coalesced and floated up to form the oil phase.

\subsection{Recycling tests}

Unlike the traditional throw-away chemical demulsifiers, the superparamagnetic feature of M-GO makes them very attractive in recycling and reusing after demulsifcation. To evaluate the potential reusability of the M-GO, recycling tests were performed under the $\mathrm{pH}$ value of 5.7. According to the aforementioned demulsification mechanism that the demulsification process was realized by M-GO strongly interacting with the asphaltene molecules at the oil-water interface, it was believed that considerable asphlatenes would be adsorbed on the surface of M-GO after demulsification. Therefore, the recycled M-GO was washed with toluene to remove the adsorbed oil and especially the asphaltenes by using the procedure as shown in Fig. 1(b). The recycling tests were repeated for 11 times. The oil concentration in the separated water and demulsification efficiency for each test were shown in Fig. 6(c). It was found that in the initial 6 cycles, the oil concentration in the separated water phase was $<20 \mathrm{mg} / \mathrm{L}$ and the corresponding demulsification efficiency was over $99.96 \%$. However, the demulsification performance started to decrease from the seventh cycle. Especially for the last 2 cycles, the oil concentration increased to over $550 \mathrm{mg} / \mathrm{L}$ and corresponding demulsification efficiency decreased to about 93.5\%. The decrease of demulsification performance of M-GO was mainly attributed to the fact that the natural surfactants such as asphaltenes and resins in the oil strongly attached on the M-GO surfaces, which could not be removed easily by toluene washing especially after multiple recycling. These results indicate that the M-GO not only has a superior demulsification performance, but also exhibits good recyclability. More importantly, the M-GO can be effectively recovered from the complex multiphase systems after demulsification by an external magnetic field. The side effects of demulsifiers on environment and/or the following oil refinery would be greatly reduced.

\section{Conclusions}

Magnetic demulsifier of M-GO was prepared and determined to be a rapid, high-efficiency and reusable demulsifier to break up the crude oil-in-water emulsion. It was found that the stability of oilin-water emulsion was greatly destroyed by adding M-GO and the demulsification process could be finished within several minutes. The oil concentration in the separated water samples was as low as $\sim 10 \mathrm{mg} / \mathrm{L}$ and the corresponding demulsification efficiency attained as high as $99.98 \%$. Such excellent demulsification performance was attributed to the fact that the small oil floccules combined with the M-GO suspending in the separated water can be eliminated by an external magnetic field. More importantly, the recycling test indicated that the M-GO can be reused for 6-7 times. The recyclable performance of M-GO not only significantly reduced the demulsification costs but also minimized the side effect of demulsifier on the environment. The non-covalent interaction analysis indicated that the strong affinity between M-GO and asphaltenes/resins was attributed to the $\pi-\pi$ and/or $\sigma-\pi$ interactions.

\section{Acknowledgements}

The authors acknowledge the financial support from the National Natural Science Foundation of China (51374195; 51574217).

\section{Appendix A. Supplementary material}

Supplementary data associated with this article can be found, in the online version, at http://dx.doi.org/10.1016/j.fuel.2016.10.066.

\section{References}

[1] Chopra S. Heavy Oils: Reservoir Characterization and Production Monitoring. Tulsa: Society of Exploration Geophysicists; 2010.

[2] Dong M, Ma S, Liu Q. Enhanced heavy oil recovery through interfacial instability: a study of chemical flooding for Brintnell heavy oil. Fuel 2009;88:1049-56.

[3] Clark K, Pasternack D. Hot water separation of bitumen from Alberta bituminous sand. Ind Eng Chem 1932;24:1410-6.

[4] Hasan S, Ghannam M, Esmail N. Heavy crude oil viscosity reduction and rheology for pipeline transportation. Fuel 2010;89:1095-100.

[5] Wang J, Hu F, Li C, Li J, Yang Y. Synthesis of dendritic polyether surfactants for demulsification. Sep Purif Technol 2010;73:349-54.

[6] Zhang Z, Xu G, Wang F, Dong S, Chen Y. Demulsification by amphiphilic dendrimer copolymers. J Colloid Interface Sci 2005;282:1-4.

[7] Daniel-David D, Le Follotec A, Pezron I, Dalmazzone C, Noik C, Barre L, et al Destabilisation of water-in-crude oil emulsions by silicone copolymer demulsifiers. Oil Gas Sci Technol 2008;63:165-73. 
[8] Feng XH, Xu ZH, Masliyah J. Biodegradable polymer for demulsification of water-in-bitumen emulsions. Energy Fuels 2008;23:451-6.

[9] Xu X, Yang J, Gao J. Effects of demulsifier structure on desalting efficiency of crude oils. Petrol Sci Technol 2006;24:673-88.

[10] Silva E, Santos D, Alves D, Barbosa M, Guimarães R, Ferreira B, et al. Demulsification of heavy crude oil emulsions using ionic liquids. Energy Fuels 2013;27:6311-5.

[11] Shannon M, Bohn P, Elimelech M, Georgiadis J, Marinas B, Mayes A. Science and technology for water purification in the coming decades. Nature 2008;452:301-10.

[12] Cheryan M, Rajagopalan N. Membrane processing of oily streams. Wastewater treatment and waste reduction. J Membr Sci 1998;151:13-28.

[13] Liang HW, Cao X, Zhang WJ, Lin TH, Zhou F, Chen LF, et al. Robust and highly efficient free-standing carbonaceous nanofiber membranes for water purification. Adv Funct Mater 2011;21:3851-8.

[14] Salahia Abdolhamid, Mohammadia Toraj, Behbahanib Reza Mosayebi, Hemmatic M. Asymmetric polyethersulfone ultrafiltration membranes for oily wastewater treatment: synthesis, characterization, ANFIS modeling, and performance. Environ Chem Eng 2015;3:170-8.

[15] Li SP, Neo JW, Ong RC, Weber W, Staudt C, Widjojo N, et al. Short and longterm performance of the thin-film composite forward osmosis (TFC-FO) hollow fiber membranes for oily wastewater purification. Ind Eng Chem Res 2014;53:14056-64.

[16] Han G, Wit JS, Chung TS. Water reclamation from emulsified oily wastewater via effective forward osmosis hollow fiber membranes under the PRO mode. Water Res 2015;81:54-63.

[17] Xue Z, Cao Y, Liu N, Feng L, Jiang L. Special wettable materials for oil/water separation. J Mater Chem A 2014;2:2445-60.

[18] Liu J, Li XC, Jia WH, Li ZY, Zhao YP, Ren SL. Demulsification of crude oil-inwater emulsions driven by graphene oxide nanosheets. Energy Fuels 2015;29:4644-53.

[19] Lu AH, Salabas E, Schüth F. Magnetic nanoparticles: synthesis, protection, functionalization, and application. Angew Chem 2007;46:1222-44.
[20] Shao M, Ning F, Zhao J, Wei M, Evans D, Duan X. Preparation of $\mathrm{Fe}_{3} \mathrm{O}_{4} @ \mathrm{SiO}_{2} @$ layered double hydroxide core-shell microspheres for magnetic separation of proteins. J Am Chem Soc 2010;134:1071-7.

[21] Liu Z, Yang H, Zhang H, Huang C, Li L. Oil-field wastewater purification by magnetic separation technique using a novel magnetic nanoparticle. Cryogenics 2012;52:699-703.

[22] Zhang W, Shi Z, Zhang F, Liu X, Jin J, Jiang L. Superhydrophobic and superoleophilic PVDF membranes for effective separation of water-in-oil emulsions with high flux. Adv Mater 2013;25:2071-6.

[23] Hummers W, Offeman R. Preparation of graphitic oxide. J Am Chem Soc 1958;80:1339.

[24] Mullins O. The modified Yen model. Energy Fuels 2010;24:2179-207.

[25] Rockenberger J, Scher E, Alivisatos A. A new nonhydrolytic single-precursor approach to surfactant-capped nanocrystals of transition metal oxides. J Am Chem Soc 1999;121:11595-6.

[26] Ni Y, Ge X, Zhang Z, Ye Q. Fabrication and characterization of the plate-shaped $\gamma-\mathrm{Fe}_{2} \mathrm{O}_{3}$ nanocrystals. Chem Mater 2002;14:1048-52.

[27] Han Q, Liu Z, Xu Y, Chen Z, Wang T, Zhang H. Growth and properties of singlecrystalline $\gamma-\mathrm{Fe}_{2} \mathrm{O}_{3}$ nanowires. J Phys Chem C 2007;111:5034-8.

[28] Peng J, Liu Q Xu ZH, Masliyah J. Synthesis of interfacially active and magnetically responsive nanoparticles for multiphase separation applications. Adv Funct Mater 2012;22:1732-40.

[29] Wu P, Xu Z. Silanation of nanostructured mesoporous magnetic particles for heavy metal recovery. Ind Eng Chem Res 2005;44:816-24.

[30] Cote L, Kim J, Tung V, Luo J, Kim F, Huang J. Graphene oxide as surfactant sheets. Pure Appl Chem 2010;83:95-110.

[31] Li D, Müller M, Gilje S, Kaner R, Wallace G. Processable aqueous dispersions of graphene nanosheets. Nat Nanotechnol 2008;3:101-5.

[32] Johnson E, Keinan S, Mori-Sanchez P, Contreras-Garcia J, Cohen A, Yang W. Revealing noncovalent interactions. J Am Chem Soc 2010;132:6498-506.

[33] Contreras-Garcia J, Yang W, Johnson ER. Analysis of hydrogen-bond interaction potentials from the electron density: integration of noncovalent interaction regions. J Phys Chem A 2011;115:12983-90. 